\title{
Pushing the envelope of minimally invasive esophagectomy
}

\author{
Chien-Hung Chiu, Yin-Kai Chao \\ Division of Thoracic Surgery, Chang Gung Memorial Hospital at Linkou, Chang Gung University, Taoyuan \\ Correspondence to: Yin-Kai Chao. Division of Thoracic and Cardiovascular Surgery, Chang Gung Memorial Hospital at Linkou, Chang Gung \\ University, No.5, Fuxing St, Guishan Dist, Taoyuan. Email: chaoyk@cgmh.org.tw. \\ Provenance: This is an invited article commission by the Academic Editor Feng Li (Department of Surgery, Competence Center of Thoracic Surgery, \\ Charité University Hospital Berlin, Berlin, Germany). \\ Comment on: Mariette C, Markar SR, Dabakuyo-Yonli TS, et al. Hybrid minimally invasive esophagectomy for esophageal cancer. N Engl J Med \\ 2019;380:152-62.
}

Submitted Aug 27, 2019. Accepted for publication Sep 04, 2019.

doi: $10.21037 /$ jtd.2019.09.42

View this article at: http://dx.doi.org/10.21037/jtd.2019.09.42

Esophageal cancer surgery-which is traditionally performed through laparotomy and thoracotomycarries significant risks in terms of morbidity and mortality (1). Over the last two decades, minimally invasive esophagectomy (MIE) has emerged as a valuable approach that may help reduce surgical trauma-ultimately minimizing complications rates. In 2012, the seminal European multicenter TIME trial demonstrated that total MIE-consisting of thoracoscopy and laparoscopy—was characterized by significantly lower rates of respiratory complications ( $9 \%$ vs. $29 \%$, respectively) and a shorter length of stay (11 vs. 14 days, respectively) compared with the traditional open approach (i.e., thoracotomy and laparotomy) (2,3). In 2018, similar results were reported in the ROBOT (Robot-assisted Thoracolaparoscopic Esophagectomy vs. Open Transthoracic Esophagectomy) trial (4). In this study, 55 patients were randomly assigned to an open three-field esophagectomy, whereas 54 patients were randomized to a robot-assisted threefield esophagectomy (with the thorax being approached robotically and the abdomen laparoscopically). The overall complication rate $(59 \%$ vs. $80 \%$, respectively), the median blood loss (400 vs. $568 \mathrm{~mL}$, respectively), and the rate of pulmonary complications ( $60 \%$ vs. $80 \%$, respectively) were all lower in the robot-assisted group. Although there were no significant intergroup differences in terms of overall and disease-free survival, pain was less severe and quality of life was higher in the robot-assisted group (4).

An important characteristic of thoracoscopic/robotic and laparoscopic surgery is the existence of a significant learning curve-with the successful adoption of both approaches requiring a significant investment of time and resources (5-7). This limitation-coupled with the well-known technical complexity of the thoracoscopic/ robotic and laparoscopic approaches-has limited the widespread adoption of MIE for patients with esophageal malignancies (7). In this context, a hybrid approachconsisting of either thoracoscopy/laparotomy or thoracotomy/laparoscopy_can represent a reasonable alternative with a less steep learning curve. Because of a lower burden in terms of pulmonary complications, avoidance of thoracotomy is generally considered a prerequisite to achieve better clinical outcomes. Therefore, hybrid MIE based on thoracoscopy and laparotomy has comparatively received more attention than the combination of thoracotomy and laparoscopy (8). Several retrospective studies have confirmed the benefits of hybrid thoracoscopic MIE over the open approach (9). However, the results of a recent randomized controlled trial conducted by the Federation de Recherche en Chirurgie (FRENCH) and French EsoGastric Tumors (FREGAT) Working Group challenged this traditional view (10). In this multicenter trial, the authors compared hybrid MIE (laparoscopic gastric mobilization and open thoracotomy) with open esophagectomy (open gastric mobilization and thoracotomy) in patients with thoracic esophageal cancer who were undergoing esophagectomy using an abdominal and right thoracic approach (Ivor Lewis procedure). The occurrence of intraoperative and postoperative complications (grade $\geq$ II according to the Calvien-Dindo 
classification) within 30 days was the primary outcome measure. Secondary endpoints included death within the first 30 post-operative days, tumor recurrence, and overall survival.

Although all patients had undergone open right thoracotomy for esophagectomy with an intrathoracic anastomosis, avoidance of laparotomy alone led to a significant reduction of major perioperative complications from $64 \%$ to $36 \%$. The incidence of major pulmonary complications similarly decreased from $30 \%$ to $18 \%$. The 30 -day (1\% and $2 \%$ in the hybrid MIE and open surgery groups, respectively) and 90 -day (4\% and $6 \%$ in the hybrid MIE and open surgery groups, respectively) mortality rates did not show significant intergroup differences. After a 3 -year follow-up, overall and disease-free survival rates did not differ significantly in the two groups, although more favorable trends were observed in patients who received hybrid MIE.

Despite the promising results in patients treated with hybrid MIE, several comments on the study findings may be offered. First, the lower rate of pulmonary complications in the hybrid MIE group did not translate either into a shorter median length of stay or a lower surgical mortality. This phenomenon may be explained by the fact that the length of incisions has a lower impact on clinical outcomes than the time required for recovering from surgery-induced changes in gastrointestinal physiology and surgical dissections. An alternative explanation is that patients in this hybrid MIE trial might have been more promptly and effectively treated upon detection of a complication. Further cost-effectiveness analyses should help cast more light on this issue. Second, we should keep in mind that clinical trials do not invariably reflect real-world practice. This issue is even more pronounced in surgical trials, which can significantly be influenced by the learning curve effect. A nationwide study conducted in Japan on an unselected cohort of 5,534 patients who had undergone McKeown esophagectomy demonstrated that MIE did not significantly reduce the 30-day rates of death and postoperative pneumonia (11). In contrast, MIE was associated with higher 30-day reoperation rates compared with open surgery. Another study conducted in the UK on an administrative cohort of 18,673 patients (with the majority being treated with the Ivor Lewis procedure) similarly failed to show the superiority of MIE over open surgery (12). When interpreting findings from clinical trials, it is also paramount to take into account the quality metrics used in the selection of surgeons and recruiting centers (i.e., 25 previous laparoscopic procedures in the hybrid MIE trial) (10).

Based on the currently available evidence from three European randomized trials, we therefore conclude that total and hybrid MIE are both significantly superior to open esophagectomy - with either technique leading to decreased complication rates when performed by experienced hands. Oncological outcomes of total and hybrid MIE are broadly similar and in line with the highest contemporary standards.

\section{Acknowledgments}

None.

\section{Footnote}

Conflicts of Interest: The authors have no conflicts of interest to declare.

Ethical Statement: The authors are accountable for all aspects of the work in ensuring that questions related to the accuracy or integrity of any part of the work are appropriately investigated and resolved.

\section{References}

1. Finks JF, Osborne NH, Birkmeyer JD. Trends in hospital volume and operative mortality for high-risk surgery. $\mathrm{N}$ Engl J Med 2011;364:2128-37.

2. Biere SS, van Berge Henegouwen MI, Maas KW, et al. Minimally invasive versus open oesophagectomy for patients with oesophageal cancer: a multicentre, open-label, randomised controlled trial. Lancet 2012;379:1887-92.

3. Straatman J, Van Der Wielen N, Cuesta MA, et al. Minimally Invasive Versus Open Esophageal Resection: Three-year Follow-up of the Previously Reported Randomized Controlled Trial the TIME Trial. Ann Surg 2017;266:232-6.

4. van der Sluis PC, van der Horst S, May AM, et al. Robot-assisted minimally invasive thoracolaparoscopic esophagectomy versus open transthoracic esophagectomy for resectable esophageal cancer: a randomized controlled trial. Ann Surg 2019;269:621-30.

5. Poon RT, Ng KK, Lam CM, et al. Learning curve for radiofrequency ablation of liver tumors: prospective analysis of initial 100 patients in a tertiary institution. Ann Surg 2004;239:441-9.

6. Chao YK, Wen YW, Chuang WY, et al. Transition from video-assisted thoracoscopic to robotic esophagectomy: a 
single surgeon's experience. Dis Esophagus 2019. [Epub ahead of print].

7. van Workum F, Stenstra MH, Berkelmans GH, et al. Learning curve and associated morbidity of minimally invasive esophagectomy: a retrospective multicenter study. Ann Surg 2019;269:88-94.

8. Burdall OC, Boddy AP, Fullick J, et al. A comparative study of survival after minimally invasive and open oesophagectomy. Surg Endosc 2015;29:431-7.

9. Bonavina L, Scolari F, Aiolfi A, et al. Early outcome of thoracoscopic and hybrid esophagectomy: propensitymatched comparative analysis. Surgery 2016;159:1073-81.
10. Mariette C, Markar SR, Dabakuyo-Yonli TS, et al. Hybrid minimally invasive esophagectomy for esophageal cancer. N Engl J Med 2019;380:152-62.

11. Takeuchi H, Miyata H, Gotoh M, et al. A risk model for esophagectomy using data of 5354 patients included in a Japanese nationwide web-based database. Ann Surg 2014;260:259-66.

12. Lazzarino AI, Nagpal K, Bottle A, et al. Open versus minimally invasive esophagectomy: trends of utilization and associated outcomes in England. Ann Surg 2010;252:292-8.
Cite this article as: Chiu CH, Chao YK. Pushing the envelope of minimally invasive esophagectomy. J Thorac Dis 2019;11(10):E171-E173. doi: 10.21037/jtd.2019.09.42 